\title{
Two classes of non-weight modules over the twisted Heisenberg-Virasoro algebra
}

\author{
Haibo Chen, Jianzhi Hanl, Yucai Su, Xiaoqing Yue
}

School of Mathematical Sciences, Tongji University, Shanghai 200092, China

E-mails: rebel1025@126.com, jzhan@tongji.edu.cn, ycsu@tongji.edu.cn, xiaoqingyue@tongji.edu.cn

Abstract: In the present paper, we construct two classes of non-weight modules $\Omega(\lambda, \alpha, \beta) \otimes$ $\operatorname{Ind}(M)$ and $\mathcal{M}(V, \Omega(\lambda, \alpha, \beta))$ over the twisted Heisenberg-Virasoro algebra, which are both associated with the modules $\Omega(\lambda, \alpha, \beta)$. We present the necessary and sufficient conditions under which modules in these two classes are irreducible and isomorphic, and also show that the irreducible

modules in these two classes are new. Finally, we construct non-weight modules $\operatorname{Ind}_{\underline{y}, \lambda}\left(\mathbb{C}_{R S}\right)$ and $\operatorname{Ind}_{\underline{z}, \lambda}\left(\mathbb{C}_{P Q}\right)$ over the twisted Heisenberg-Virasoro algebra and then apply the established results to give irreducible conditions for $\operatorname{Ind}_{\underline{y}, \lambda}\left(\mathbb{C}_{R S}\right)$ and $\operatorname{Ind}_{\underline{z}, \lambda}\left(\mathbb{C}_{P Q}\right)$.

Key words: twisted Heisenberg-Virasoro algebra, non-weight module, irreducible module.

Mathematics Subject Classification (2010): 17B10, 17B65, 17B68.

\section{Introduction}

The well-known twisted Heisenberg-Virasoro algebra $\mathcal{H}$, initially studied by Arbarello et al. in [1], is the universal central extension of the Lie algebra $\bar{L}$ of differential operators on a circle of order at most one:

$$
\bar{L}:=\left\{f(t) \frac{d}{d t}+g(t) \mid f(t), g(t) \in \mathbb{C}\left[t, t^{-1}\right]\right\} .
$$

To be more precise, $\mathcal{H}$ is an infinite dimensional complex Lie algebra with basis $\left\{L_{m}, I_{m}, C_{i} \mid\right.$ $m \in \mathbb{Z}, i=1,2,3\}$ subject to the following Lie brackets:

$$
\begin{aligned}
& {\left[L_{m}, L_{n}\right]=(n-m) L_{m+n}+\delta_{m+n, 0} \frac{m^{3}-m}{12} C_{1},} \\
& {\left[L_{m}, I_{n}\right]=n I_{m+n}+\delta_{m+n, 0}\left(m^{2}+m\right) C_{2},} \\
& {\left[I_{m}, I_{n}\right]=n \delta_{m+n, 0} C_{3},} \\
& {\left[\mathcal{H}, C_{1}\right]=\left[\mathcal{H}, C_{2}\right]=\left[\mathcal{H}, C_{3}\right]=0 .}
\end{aligned}
$$

Clearly, the subspaces spanned by $\left\{I_{m}, C_{3} \mid 0 \neq m \in \mathbb{Z}\right\}$ and by $\left\{L_{m}, C_{1} \mid m \in \mathbb{Z}\right\}$ are respectively the Heisenberg algebra and the Virasoro algebra. Notice that the center of $\mathcal{H}$ is spanned by $\left\{C_{0}:=I_{0}, C_{i} \mid i=1,2,3\right\}$. Moreover, the twisted Heisenberg-Virasoro algebra has a triangular decomposition:

$$
\mathcal{H}=\mathcal{H}_{-} \oplus \mathfrak{h} \oplus \mathcal{H}_{+},
$$

Correspondence: J. Han (jzhan@tongji.edu.cn) 
where $\mathfrak{h}=\operatorname{span}_{\mathbb{C}}\left\{L_{0}, C_{i} \mid i=0,1,2,3\right\}$ and

$$
\mathcal{H}_{-}=\operatorname{span}_{\mathbb{C}}\left\{L_{-m}, I_{-m} \mid m \in \mathbb{N}\right\}, \mathcal{H}_{+}=\operatorname{span}_{\mathbb{C}}\left\{L_{m}, I_{m} \mid m \in \mathbb{N}\right\}
$$

The twisted Heisenberg-Virasoro algebra is one of the most important Lie algebras both in mathematics and in mathematical physics, whose structure theory has extensively studied (see, e.g., [7, 10,20]).

A fundamental problem in the representation theory of the twisted Heisenberg-Virasoro algebra is to classify all its irreducible modules. In fact, the theory of weight modules with all weight subspaces being finite dimensional (namely, Harish-Chandra-modules) is welldeveloped. Irreducible weight modules over $\mathcal{H}$ with a nontrivial finite dimensional weight subspace were proved to be Harish-Chandra modules [22]. And irreducible Harish-Chandra $\mathcal{H}$-modules were classified in [15], each of which was shown to be either the highest (or lowest) weight module, or the module of intermediate series, consistent with the well-known result for Virasoro algebra [16. While weight modules with an infinite dimensional weight subspace were also studied (see [6, 19]).

Non-weight modules constitute the other important ingredients of the representation theory of $\mathcal{H}$, the study of which is definitely necessary and became popular in the last few years. A large class of new non-weight irreducible $\mathcal{H}$-modules were constructed in [3], which includes the highest weight modules and Whittaker modules. Non-weight $\mathcal{H}$-modules whose restriction to the universal enveloping algebra of the degree-0 part (modulo center) are free of rank 1 were studied in [4] (see also [8]). While by twisting the weight modules, the authors [6] obtained a family of new non-weight irreducible $\mathcal{H}$-modules. However, the theory of non-weight $\mathcal{H}$-modules is far more from being well-developed.

As a continuation of [6], we still study the representation theory of $\mathcal{H}$ in this paper. But we shall be concerned with non-weight $\mathcal{H}$-modules. To be more precisely, we construct two classes of new irreducible non-weight $\mathcal{H}$-modules in the present paper, which are both related to the modules $\Omega(\lambda, \alpha, \beta)$ (see [4]). It is well known that an important way of constructing modules is to consider the linear tensor product of two modules, see for instance, [5, 23, 25] for such modules over the Virasoro algebra. One class of non-weight modules is constructed by considering the tensor product module and the other class also stems from the linear tensor product of two modules but on which different actions are given.

We briefly give a summary of the paper below. In Section 2, we recall some known modules and construct a class of non-weight modules $\mathcal{M}(V, \Omega(\lambda, \alpha, \beta))$ over the twisted Heisenberg-Virasoro algebra. Section 3 is devoted to studying the irreducibilities of tensor product modules $\Omega(\lambda, \alpha, \beta) \otimes \operatorname{Ind}(M)$ and the reducibilities of $\mathcal{H}$-modules $\mathcal{M}(V, \Omega(\lambda, \alpha, \beta))$. We prove that $\mathcal{M}(V, \Omega(\lambda, \alpha, \beta))$ is reducible if and only if $V$ is a certain one-dimensional $\overline{\mathcal{H}}_{r, d}$-module. In Section 4, we give the necessary and sufficient conditions for two irreducible $\mathcal{H}$-modules $\Omega\left(\lambda_{1}, \alpha_{1}, \beta_{1}\right) \otimes \operatorname{Ind}\left(M_{1}\right)$ and $\Omega\left(\lambda_{2}, \alpha_{2}, \beta_{2}\right) \otimes \operatorname{Ind}\left(M_{2}\right)$ to be isomorphic. 
Moreover, we determine the isomorphism classes of the other class of non-weight $\mathcal{H}$-modules $\mathcal{M}(V, \Omega(\lambda, \alpha, \beta))$. In section 5 , we give some practical examples of irreducible modules of $\Omega(\lambda, \alpha, \beta) \otimes \operatorname{Ind}(M)$, and present the irreducibilities of modules $\operatorname{Ind}_{\underline{y}, \lambda}\left(\mathbb{C}_{R S}\right)$ and $\operatorname{Ind}_{\underline{z}, \lambda}\left(\mathbb{C}_{P Q}\right)$.

At last, we show that irreducible $\mathcal{H}$-modules in these two classes are new by showing they are not isomorphic to each other or the known ones. The main results of this paper are summarized in Theorems 3.1, 3.3, 4.1, 4.4, 6.2 and 6.3,

Throughout this paper, we respectively denote by $\mathbb{C}, \mathbb{C}^{*}, \mathbb{Z}, \mathbb{Z}_{+}$and $\mathbb{N}$ the sets of complex numbers, nonzero complex numbers, integers, nonnegative integers and positive integers, and use $\mathcal{U}(\mathfrak{a})$ to denote the universal enveloping algebra of $\mathfrak{a}$. All vector spaces are assumed to be over $\mathbb{C}$.

\section{Some non-weight modules}

\subsection{Known modules $\Omega(\lambda, \alpha, \beta)$ and $\operatorname{Ind}(M)$}

For $\lambda \in \mathbb{C}^{*}, \alpha, \beta \in \mathbb{C}$, we recall the non-weight module

$$
\Omega(\lambda, \alpha, \beta):=\mathbb{C}[t]
$$

with the action of $\mathcal{H}$ defined, for $i=1,2,3, f(t) \in \mathbb{C}[t]$ and $m \in \mathbb{Z}$, by

$$
L_{m} \cdot f(t)=\lambda^{m}(t-m \alpha) f(t-m), I_{m} \cdot f(t)=\lambda^{m} \beta f(t-m), C_{i} \cdot f(t)=0 .
$$

Then $\Omega(\lambda, \alpha, \beta)$ is irreducible if and only if $\alpha \in \mathbb{C}^{*}$ or $\beta \in \mathbb{C}^{*}$ (see [4]). Notice that this module reduces to a Virasoro module if $\beta=0$ (see [13]).

Now let us recall a large class of irreducible modules for the twisted Heisenberg-Virasoro algebra, which includes the known irreducible modules such as highest weight modules and Whittaker modules. For any $e \in \mathbb{Z}_{+}$, denote by $\mathcal{H}_{e}$ the subalgebra

$$
\sum_{m \in \mathbb{Z}_{+}}\left(\mathbb{C} L_{m} \oplus \mathbb{C} I_{m-e}\right) \oplus \mathbb{C} C_{1} \oplus \mathbb{C} C_{2} \oplus \mathbb{C} C_{3}
$$

Take $M\left(c_{0}, c_{1}, c_{2}, c_{3}\right)$ to be an irreducible $\mathcal{H}_{e}$-module such that $I_{0}, C_{1}, C_{2}$ and $C_{3}$ act on it as scalars $c_{0}, c_{1}, c_{2}, c_{3}$ respectively. For convenience, we briefly denote $M\left(c_{0}, c_{1}, c_{2}, c_{3}\right)$ by $M$ and form the induced $\mathcal{H}$-module

$$
\operatorname{Ind}(M):=\mathcal{U}(\mathcal{H}) \otimes_{\mathcal{U}\left(\mathcal{H}_{e}\right)} M
$$

The following theorem is obtained in [3].

Theorem 2.1. Let $e \in \mathbb{Z}_{+}$and $M$ be an irreducible $\mathcal{H}_{e}$-module with $c_{3}=0$. Assume there exists $k \in \mathbb{Z}_{+}$such that 
(1) $\begin{cases}\text { the action of } I_{k} \text { on } M \text { is injective } & \text { if } k \neq 0, \\ c_{0}+(n-1) c_{2} \neq 0 \text { for all } n \in \mathbb{Z} \backslash\{0\} & \text { if } k=0,\end{cases}$

(2) $I_{n} M=L_{m} M=0$ for all $n>k$ and $m>k+e$.

Then

(i) $\operatorname{Ind}(M)$ is an irreducible $\mathcal{H}$-module;

(ii) the actions of $I_{n}, L_{m}$ on $\operatorname{Ind}(M)$ for all $n>k$ and $m>k+e$ are locally nilpotent.

\subsection{Construction of $\mathcal{M}(V, \Omega(\lambda, \alpha, \beta))$}

For $d \in\{0,1\}, r \in \mathbb{Z}_{+}$, denote by $\mathcal{H}_{r, d}$ the Lie subalgebra of $\mathcal{H}_{+, d}=\operatorname{span}_{\mathbb{C}}\left\{L_{i}, I_{j} \mid i \geq\right.$ $0, j \geq d\}$ generated by $L_{i}, I_{j}$ for all $i>r, j>r+d$. Now we write $\overline{\mathcal{H}}_{r, d}$ the quotient algebra $\mathcal{H}_{+, d} / \mathcal{H}_{r, d}$, and $\bar{L}_{i}, \bar{I}_{i+d}$ the respective images of $L_{i}, I_{i+d}$ in $\overline{\mathcal{H}}_{r, d}$. Let $V$ be an $\overline{\mathcal{H}}_{r, d}$-module. For any $\lambda, \alpha, \beta \in \mathbb{C}$, define an $\mathcal{H}$-action on the vector space $\mathcal{M}(V, \Omega(\lambda, \alpha, \beta)):=V \otimes \mathbb{C}[t]$ as follows

$$
\begin{aligned}
& L_{m}(v \otimes f(t))=v \otimes \lambda^{m}(t-m \alpha) f(t-m)+\sum_{i=0}^{r}\left(\frac{m^{i+1}}{(i+1) !} \bar{L}_{i}\right) v \otimes \lambda^{m} f(t-m), \\
& I_{m}(v \otimes f(t))=\sum_{i=0}^{r}\left(\frac{m^{i+d}}{(i+d) !} \bar{I}_{i+d}\right) v \otimes \lambda^{m} \beta f(t-m), \\
& C_{i}(v \otimes f(t))=0 \quad \text { for } i \in\{1,2,3\}, m \in \mathbb{Z}, v \in V, f(t) \in \mathbb{C}[t] .
\end{aligned}
$$

Proposition 2.2. Let $d \in\{0,1\}, r \in \mathbb{Z}_{+}$and $V$ be an $\overline{\mathcal{H}}_{r, d}$ module. Then $\mathcal{M}(V, \Omega(\lambda, \alpha, \beta))$ is a non-weight $\mathcal{H}$-module under the actions given in (2.3) $-(2.5)$.

Proof. Define a series of operators $x_{m}$ on $\mathbb{C}[t]$ as follows:

$$
x_{m} f(t)=\lambda^{m} f(t-m) \quad \text { for } m \in \mathbb{Z} \text { and } f(t) \in \mathbb{C}[t] .
$$

Then $I_{n} x_{m} f(t)=x_{m} I_{n} f(t)=I_{m+n} f(t)$ for any $m, n \in \mathbb{Z}$. It follows from [14, Section 3] that the relation $L_{m} L_{n}-L_{n} L_{m}=(n-m) L_{m+n}$ holds on $\mathcal{M}(V, \Omega(\lambda, \alpha, \beta))$. By (2.3)-(2.5), we have

$$
\begin{aligned}
& \left(L_{m} I_{n}-I_{n} L_{m}\right)(v \otimes f(t)) \\
= & \sum_{i=0}^{r}\left(\frac{n^{i+d}}{(i+d) !} \bar{I}_{i+d}\right) v \otimes\left(L_{m} I_{n} f(t)\right) \\
& +\sum_{i=0}^{r}\left(\frac{m^{i+1}}{(i+1) !} \bar{L}_{i}\right) \sum_{i=0}^{r}\left(\frac{n^{i+d}}{(i+d) !} \bar{I}_{i+d}\right) v \otimes\left(x_{m} I_{n} f(t)\right)
\end{aligned}
$$




$$
\begin{aligned}
& -\sum_{i=0}^{r}\left(\frac{n^{i+d}}{(i+d) !} \bar{I}_{i+d}\right) v \otimes\left(I_{n} L_{m} f(t)\right) \\
& -\sum_{i=0}^{r}\left(\frac{n^{i+d}}{(i+d) !} \bar{I}_{i+d}\right) \sum_{i=0}^{r}\left(\frac{m^{i+1}}{(i+1) !} \bar{L}_{i}\right) v \otimes\left(I_{n} x_{m} f(t)\right) \\
& =n \sum_{i=0}^{r}\left(\frac{n^{i+d}}{(i+d) !} \bar{I}_{i+d}\right) v \otimes\left(I_{m+n} f(t)\right) \\
& +\sum_{i=0}^{r}\left(\frac{m^{i+1}}{(i+1) !} \bar{L}_{i}\right) \sum_{j=0}^{r}\left(\frac{n^{j+d}}{(j+d) !} \bar{I}_{j+d}\right) v \otimes\left(I_{m+n} f(t)\right) \\
& -\sum_{j=0}^{r}\left(\frac{n^{j+d}}{(j+d) !} \bar{I}_{j+d}\right) \sum_{i=0}^{r}\left(\frac{m^{i+1}}{(i+1) !} \bar{L}_{i}\right) v \otimes\left(I_{m+n} f(t)\right) \\
& =n \sum_{i=0}^{r}\left(\frac{n^{i+d}}{(i+d) !} \bar{I}_{i+d}\right) v \otimes\left(I_{m+n} f(t)\right) \\
& +\sum_{i, j=0}^{r}\left(\frac{m^{i+1} n^{j+d}}{(i+1) !(j+d) !}\left(\bar{L}_{i} \bar{I}_{j+d}-\bar{I}_{j+d} \bar{L}_{i}\right)\right) v \otimes\left(I_{m+n} f(t)\right) \\
& =n \sum_{i=0}^{r} \sum_{j=\delta_{d, 0}}^{i+1}\left(\frac{m^{i+1-j} n^{j+d-1}}{(i+1-j) !(j+d-1) !} \bar{I}_{i+d}\right) v \otimes\left(I_{m+n} f(t)\right) \\
& =n \sum_{i=0}^{r}\left(\frac{(m+n)^{i+d}}{(i+d) !} \bar{I}_{i+d}\right) v \otimes\left(I_{m+n} f(t)\right)=n I_{m+n}(v \otimes f(t)) .
\end{aligned}
$$

That is, $L_{m} I_{n}-I_{n} L_{m}=n I_{n+m}$ holds on $\mathcal{M}(V, \Omega(\lambda, \alpha, \beta))$. Finally, the relation $I_{m} I_{n}-I_{n} I_{m}=$ 0 on $\mathcal{M}(V, \Omega(\lambda, \alpha, \beta))$ is trivial. Thus, the actions (2.3) - 2.5) make $\mathcal{M}(V, \Omega(\lambda, \alpha, \beta))$ into a non-weight $\mathcal{H}$-module.

Remark 2.3. Let $d \in\{0,1\}, r \in \mathbb{Z}_{+}$and $V$ be an irreducible $\overline{\mathcal{H}}_{r, d}$-module.

(1) $V$ must be infinite dimensional if $\operatorname{dim} V>1$, since any irreducible finite dimensional module over the solvable Lie algebra $\overline{\mathcal{H}}_{r, d}$ is one-dimensional by Lie's Theorem.

(2) Consider now $V=\mathbb{C} v$ is one-dimensional. Then $\bar{L}_{i} v=\bar{I}_{i} v=0$ for any $i \in \mathbb{N}$, $\bar{L}_{0} v=\sigma v, \bar{I}_{0} v=\tau v$ for some $\sigma, \tau \in \mathbb{C}$. In this case $V$ is denoted by $V_{\sigma, \tau}$ and it is clear that

$$
\mathcal{M}\left(V_{\sigma, \tau}, \Omega(\lambda, \alpha, \beta)\right) \cong \Omega\left(\lambda, \alpha-\sigma, \delta_{d, 0} \beta \tau\right) \text { for any } \lambda \in \mathbb{C}^{*} \text { and } \alpha, \beta \in \mathbb{C},
$$

where $\delta_{d, 0}$ is the Kronecker delta.

(3) Note that if $\bar{I}_{j+d} V=0$ for all $0 \leq j \leq r$, then $\mathcal{M}(V, \Omega(\lambda, \alpha, \beta))$ reduces to a module over the Virasoro algebra. So until further notice we always assume that $\bar{I}_{j+d} V \neq 0$ for some $0 \leq j \leq r$. Choose such $r^{\prime}$ to be maximal such that $\bar{I}_{r^{\prime}+d} V \neq 0$. Then $\bar{I}_{r^{\prime}+d} i s$ a linear isomorphism by [6, Lemma 3.1] if $V$ is irreducible. 
For $\lambda, \alpha, \beta \in \mathbb{C}$, the $\mathcal{H}$-module $A(\lambda, \alpha, \beta)$ of intermediate series has a $\mathbb{C}$-basis $\left\{v_{i} \mid i \in \mathbb{Z}\right\}$ with trivial central actions and

$$
L_{m} v_{n}=(\lambda+n+m \alpha) v_{m+n}, I_{m} v_{n}=\beta v_{m+n} \quad \text { for } m, n \in \mathbb{Z} .
$$

Note that $A(\lambda, \alpha, \beta)$ is reducible if and only if $\lambda \in \mathbb{Z}, \alpha \in\{0,1\}$ and $\beta=0$ (see [9,11]). Let $d \in\{0,1\}, r \in \mathbb{Z}_{+}$and $V$ be an $\overline{\mathcal{H}}_{r, d}$-module. Define the action of $\mathcal{H}$ on $\mathcal{M}(V, A(\lambda, \alpha, \beta)):=$ $V \otimes A(\lambda, \alpha, \beta)$ as follows

$$
\begin{aligned}
& L_{m}\left(u \otimes v_{n}\right)=\left(n+\lambda+\alpha m+\sum_{i=0}^{r}\left(\frac{m^{i+1}}{(i+1) !} \bar{L}_{i}\right)\right) u \otimes v_{m+n}, \\
& I_{m}\left(u \otimes v_{n}\right)=\sum_{i=0}^{r}\left(\frac{\beta m^{i+d}}{(i+d) !} \bar{I}_{i+d}\right) u \otimes v_{m+n}, \\
& C_{1}\left(u \otimes v_{n}\right)=C_{2}\left(u \otimes v_{n}\right)=C_{3}\left(u \otimes v_{n}\right)=0,
\end{aligned}
$$

where $m, n \in \mathbb{Z}$ and $u \in V$. Then one can check that under the given actions as above, $\mathcal{M}(V, A(\lambda, \alpha, \beta))$ becomes a weight $\mathcal{H}$-module.

Definition 2.4. Let $V, W$ be two $\overline{\mathcal{H}}_{r, d}$-modules and $\alpha \in \mathbb{C}$. Denote by $V^{\alpha}$ the $\overline{\mathcal{H}}_{r, d}$-module obtained from $V$ by modifying the $\bar{L}_{0}$-action as $\bar{L}_{0}-\alpha \operatorname{id}_{V}$. An invertible linear map $\psi: V \rightarrow$ $W$ is called an $\alpha$-isomorphism if $\psi\left(\bar{L}_{i} v\right)=\bar{L}_{i} \psi(v)$ and $\psi\left(\bar{I}_{i+d} v\right)=\alpha \bar{I}_{i+d} \psi(v)$ for any $i \in \mathbb{Z}$.

It follows from the similar proof of [6, Theorem 4.1] that we have the following result.

Theorem 2.5. Let $d_{i} \in\{0,1\}, r_{i} \in \mathbb{Z}_{+}, \lambda_{i}, \alpha_{i}, 0 \neq \beta_{i} \in \mathbb{C}$ and $V_{i}$ be an irreducible $\overline{\mathcal{H}}_{r_{i}, d_{i}}{ }^{-}$ module for $i=1,2$. Then $\mathcal{M}\left(V_{1}, A\left(\lambda_{1}, \alpha_{1}, \beta_{1}\right)\right)$ and $\mathcal{M}\left(V_{2}, A\left(\lambda_{2}, \alpha_{2}, \beta_{2}\right)\right)$ are isomorphic as $\mathcal{H}$-modules if and only if $\lambda_{1}-\lambda_{2} \in \mathbb{Z}, d_{1}=d_{2}$ and $V_{1}^{\alpha_{1}} \cong V_{2}^{\alpha_{2}}$ are $\beta_{1}^{-1} \beta_{2}$-isomorphic as $\overline{\mathcal{H}}_{\max \left\{r_{1}, r_{2}\right\}, d_{1}}$-modules.

\section{Irreducibilities}

Theorem 3.1. Let $(\lambda, \alpha) \in\left(\mathbb{C}^{*}\right)^{2}$ or $(\lambda, \beta) \in\left(\mathbb{C}^{*}\right)^{2}$. Assume $\operatorname{Ind}(M)$ is an $\mathcal{H}$-module defined by (2.2) for which $M$ satisfies the conditions in Theorem [2.1. Then the tensor product $\Omega(\lambda, \alpha, \beta) \otimes \operatorname{Ind}(M)$ of $\mathcal{H}$-modules $\Omega(\lambda, \alpha, \beta)$ and $\operatorname{Ind}(M)$ is an irreducible $\mathcal{H}$-module.

Proof. For any $v \in \operatorname{Ind}(M)$, there exists $K(v) \in \mathbb{Z}_{+}$such that $I_{m} \cdot v=L_{m} \cdot v=0$ for all $m \geq K(v)$ by Theorem 2.1. Suppose $P$ is a nonzero submodule of $\Omega(\lambda, \alpha, \beta) \otimes \operatorname{Ind}(M)$. Choose a nonzero

$$
w=\sum_{i=0}^{n} t^{i} \otimes v_{i} \in P \text { with } 0 \neq v_{n} \in \operatorname{Ind}(M) \text { and } n \in \mathbb{Z}_{+} \text {is minimal. }
$$


The case for $\alpha \in \mathbb{C}^{*}$ was proved in [23, Theorem 1], thus we only need to consider the case for $\beta \in \mathbb{C}^{*}$.

Claim 1. $n=0$.

Let $K=\max \left\{K\left(v_{i}\right) \mid i=0,1, \ldots, n\right\}$. Then we have

$$
\lambda^{-m} I_{m} \cdot w=\sum_{i=0}^{n} \beta(t-m)^{i} \otimes v_{i} \in P \text { for } m \geq K
$$

Note that the right-hand side of the above can also be written as

$$
\sum_{i=0}^{n} m^{i} w_{i} \in P
$$

for some $w_{i} \in \Omega(\lambda, \alpha, \beta) \otimes \operatorname{Ind}(M)$ (independent of the choice of $m$ ) with $w_{n}=\beta(-1)^{n} \otimes v_{n} \neq$ 0 . It follows from that $w_{n} \in P$. Thus, $n$ must be zero by its minimality, proving the claim.

To complete the proof, it suffices to show the following claim.

Claim 2. $P=\Omega(\lambda, \alpha, \beta) \otimes \operatorname{Ind}(M)$.

By Claim 1, we have $1 \otimes v_{0} \in P$ for some nonzero $v_{0} \in \operatorname{Ind}(M)$. Using

$$
\begin{aligned}
L_{m} \cdot\left(t^{k} \otimes v_{0}\right) & =\left(\lambda^{m}(t-m a)(t-m)^{k}\right) \otimes v_{0} \\
& =\lambda^{m}(t-m)^{k+1} \otimes v_{0}-\lambda^{m} m(\alpha-1)(t-m)^{k} \otimes v_{0}
\end{aligned}
$$

for $m \geq K\left(v_{0}\right), k \in \mathbb{Z}_{+}$and by induction on $k$, we deduce that $t^{k} \otimes v_{0} \in P$ for $k \in \mathbb{Z}_{+}$, i.e., $\Omega(\lambda, \alpha, \beta) \otimes v_{0} \subseteq P$. It follows that $\Omega(\lambda, \alpha, \beta) \otimes \mathcal{U}(\mathcal{H}) v_{0} \subseteq P$. Thus, $P=\Omega(\lambda, \alpha, \beta) \otimes \operatorname{Ind}(M)$, since the nonzero $\mathcal{H}$-submodule $\mathcal{U}(\mathcal{H}) v_{0}$ of $\operatorname{Ind}(M)$ generated by $v_{0}$ is equal to $\operatorname{Ind}(M)$ by the irreducibility of $\operatorname{Ind}(M)$.

Now we describe the following two examples of the modules in Theorem [3.1, which will be discussed in detail in Section 6 .

Example 3.2. (i) Let $h \in \mathbb{C}, \underline{d}=\left(d_{0}, d_{1}, d_{2}, d_{3}\right) \in \mathbb{C}^{4}$ with $d_{3}=0$. Assume $J_{1}$ is the left ideal of $\mathcal{U}\left(\mathfrak{h} \oplus \mathcal{H}_{+}\right)$generated by $L_{m}, I_{m}, L_{0}-h$ and $C_{i}-d_{i}$ for $i=0,1,2,3, m \in \mathbb{Z}_{+}$. Denote $\bar{M}:=\mathcal{U}\left(\mathfrak{h} \oplus \mathcal{H}_{+}\right) / J_{1}$. Then $V=\operatorname{Ind}(\bar{M})$ is the classical Verma module (see, e.g., [2,21]). By Theorem 2.1 (cf. [2, Theorem 1]), we obtain that if $d_{0}+(n-1) d_{2} \neq 0$ for $n \in \mathbb{Z} \backslash\{0\}$, then $V$ is both an irreducible $\mathcal{H}$-module and a locally nilpotent module over $\mathcal{H}_{+}$. From Theorem 3.1, we obtain that $\Omega(\lambda, \alpha, \beta) \otimes V$ is an irreducible $\mathcal{H}$-module if $d_{0}+(n-1) d_{2} \neq 0$ for $n \in \mathbb{Z} \backslash\{0\}$ and either $(\lambda, \alpha) \in\left(\mathbb{C}^{*}\right)^{2}$ or $(\lambda, \beta) \in\left(\mathbb{C}^{*}\right)^{2}$.

(ii) Let $\left(\lambda_{1}, \lambda_{2}, \mu_{1}\right) \in \mathbb{C}^{3}, \underline{e}=\left(e_{0}, e_{1}, e_{2}, e_{3}\right) \in \mathbb{C}^{4}$ with $e_{3}=0$. Assume that $J_{2}$ is the left ideal of $\mathcal{U}\left(\mathcal{H}_{+}\right)$generated by $L_{1}-\lambda_{1}, L_{2}-\lambda_{2}, L_{3}, L_{4}, \ldots, I_{1}-\mu_{1}, I_{2}, I_{3}, \ldots, C_{i}-e_{i}$ for $i=0,1,2,3$. Denote $\tilde{M}:=\mathcal{U}\left(\mathcal{H}_{+}\right) / J_{2}$. Then $V=\operatorname{Ind}(\tilde{M})$ is the classical Whittaker 
module (see, e.g., [3, 12]). By Theorem 2.1 (cf. [3, Example 10]), we obtain that if $e_{0}+(n-1) e_{2} \neq 0$ for all $n \in \mathbb{Z} \backslash\{0\}$ and $\mu_{1} \neq 0$, then $V$ is both an irreducible $\mathcal{H}$-module and a locally nilpotent module over $\mathcal{H}_{+}^{(2)}=\operatorname{span}_{\mathbb{C}}\left\{L_{m}, I_{m} \mid m>2\right\}$. From Theorem 3.1, we obtain that $\Omega(\lambda, \alpha, \beta) \otimes V$ is an irreducible $\mathcal{H}$-module if $e_{0}+(n-1) e_{2} \neq 0$ for all $n \in \mathbb{Z} \backslash\{0\}$ and either $\left(\lambda, \alpha, \mu_{1}\right) \in\left(\mathbb{C}^{*}\right)^{3}$ or $\left(\lambda, \beta, \mu_{1}\right) \in\left(\mathbb{C}^{*}\right)^{3}$.

Next we are going to characterise the reducibility of $\mathcal{M}(V, \Omega(\lambda, \alpha, \beta))$. For any $m \in$ $\mathbb{Z}_{+}, n \in \mathbb{Z}$, denote

$$
J_{n}^{0}=1 \text { and } J_{n}^{m}=\prod_{j=n+1}^{n+m}(t-j) \text { for } m>0 .
$$

Note that $\left\{J_{n}^{m} \mid m \in \mathbb{Z}_{+}\right\}$forms a basis of $\Omega(\lambda, \alpha, \beta)$ for any $n \in \mathbb{Z}$. By the action of $\mathcal{H}$ on $\Omega(\lambda, \alpha, \beta)$, it is easy to check that

$$
L_{m} J_{n}^{k}=\lambda^{m}(t-m \alpha) J_{m+n}^{k} \text { and } I_{m} J_{n}^{k}=\lambda^{m} \beta J_{m+n}^{k} \quad \text { for } m, n \in \mathbb{Z}, k \in \mathbb{Z}_{+} .
$$

Now we are ready to state the other main result of this section.

Theorem 3.3. Let $\lambda \in \mathbb{C}^{*}$ and $V$ be an irreducible $\overline{\mathcal{H}}_{r, d}$-module. Then $\mathcal{M}(V, \Omega(\lambda, \alpha, \beta))$ is reducible if and only if $V \cong V_{\alpha, \delta_{d, 0} \tau}$ for some $\tau \in \mathbb{C}$ such that $\delta_{d, 0} \beta \tau=0$.

Proof. Consider first that $V$ is finite dimensional. Then $V \cong V_{\sigma, \tau}$ for some $\sigma, \tau \in \mathbb{C}$ and $\mathcal{M}\left(V_{\sigma, \tau}, \Omega(\lambda, \alpha, \beta)\right) \cong \Omega\left(\lambda, \alpha-\sigma, \delta_{d, 0} \beta \tau\right)$ by Remark 2.3(2). But we know that $\Omega(\lambda, \alpha-$ $\left.\sigma, \delta_{d, 0} \beta \tau\right)$ is reducible if and only if $\alpha=\sigma$ and $\delta_{d, 0} \beta \tau=0$. So in this case the statement is true.

Assume that $V$ is infinite dimensional. To complete the proof, it suffices to show that $\mathcal{M}(V, \Omega(\lambda, \alpha, \beta))$ is irreducible. For this, let $M$ be a nonzero submodule of $\mathcal{M}(V, \Omega(\lambda, \alpha, \beta))$. Without loss of generality, we may assume that $\lambda=1$. If $\beta=0$, then $\mathcal{M}(V, \Omega(\lambda, \alpha, \beta))$ reduces to a module over the Virasoro algebra, which is irreducible by [14, Theorem 3.2].

Now consider the case $\beta \neq 0$. Let $r^{\prime}$ be the nonnegative integer as in Remark 2.3)(3) and $u=\sum_{m=0}^{p} v_{m} \otimes J_{0}^{m}$ a nonzero element in $M$ with $v_{p} \neq 0$. It follows from (2.4) and the second relation in (3.1) that

$$
I_{n}\left(v_{m} \otimes J_{0}^{m}\right)=\sum_{i=0}^{r^{\prime}}\left(\frac{n^{i+d}}{(i+d) !} \bar{I}_{i+d}\right) v_{m} \otimes \beta J_{n}^{m} \quad \text { for } n \in \mathbb{Z}, m \in \mathbb{Z}_{+} .
$$

Then we can check that

$$
\begin{aligned}
I_{k} I_{n-k}\left(v_{m} \otimes J_{0}^{m}\right) & =I_{k}\left(\sum_{i=0}^{r^{\prime}}\left(\frac{(n-k)^{i+d}}{(i+d) !} \bar{I}_{i+d}\right) v_{m} \otimes \beta J_{n-k}^{m}\right) \\
& =\sum_{i=0}^{r^{\prime}}\left(\frac{k^{i+d}}{(i+d) !} \bar{I}_{i+d}\right) \sum_{i=0}^{r^{\prime}}\left(\frac{(n-k)^{i+d}}{(i+d) !} \bar{I}_{i+d}\right) v_{m} \otimes \beta^{2} J_{n}^{m} .
\end{aligned}
$$


Since $k$ is arbitrary, we can view $k$ as a variable. Observe that the coefficient of $k^{2 r^{\prime}+2 d}$ in $I_{k} I_{n-k} u$ is

$$
\frac{\beta^{2}}{\left(\left(r^{\prime}+d\right) !\right)^{2}} \sum_{m=0}^{p} \bar{I}_{r^{\prime}+d}^{2} v_{m} \otimes J_{n}^{m} \in M \quad \text { for } n \in \mathbb{Z}
$$

Similarly, viewing $n$ as a variable we get $\bar{I}_{r^{\prime}+d}^{2} v_{p} \otimes 1 \in M$. Set $v=\bar{I}_{r^{\prime}+d}^{2} v_{p}$. Now by $L_{0}^{n}(v \otimes 1)=v \otimes t^{n}$ for $n \in \mathbb{Z}$ and the injectivity of $\bar{I}_{r^{\prime}+d}$ we see that $v \otimes \Omega(\lambda, \alpha, \beta)$ is nonzero subspace of $M$. It follows from

$$
\begin{gathered}
M \ni L_{n}\left(v \otimes J_{k-n}^{m}\right)=v \otimes(t-n \alpha) J_{k}^{m}+\sum_{i=0}^{r^{\prime}}\left(\frac{n^{i+1}}{(i+1) !} \bar{L}_{i}\right) v \otimes J_{k}^{m} \\
\text { and } \quad M \ni I_{n}\left(v \otimes J_{k-n}^{m}\right)=\sum_{i=0}^{r^{\prime}}\left(\frac{n^{i+d}}{(i+d) !} \bar{I}_{i+d}\right) v \otimes \beta J_{k}^{m}
\end{gathered}
$$

that both $\sum_{i=0}^{r^{\prime}}\left(\frac{n^{i+1}}{(i+1) !} \bar{L}_{i}\right) v \otimes J_{k}^{m}$ and $\sum_{i=0}^{r^{\prime}}\left(\frac{n^{i+d}}{(i+d) !} \bar{I}_{i+d}\right) v \otimes J_{k}^{m}$ lie in $M$ for $k, m \in \mathbb{Z}, n \in \mathbb{Z}_{+}$. In particular, $\bar{L}_{i} v \otimes \Omega(\lambda, \alpha, \beta) \in M$ and $\bar{I}_{i+d} v \otimes \Omega(\lambda, \alpha, \beta) \in M$ for $i=0,1, \ldots, r^{\prime}$. Then we must have $M=\mathcal{M}(V, \Omega(\lambda, \alpha, \beta))$, since $V$ is irreducible. This shows the irreducibility of $\mathcal{M}(V, \Omega(\lambda, \alpha, \beta))$, completing the proof of Theorem 3.3 .

\section{Isomorphism classes}

Theorem 4.1. Let $\alpha_{1}, \beta_{1} \in \mathbb{C},\left(\lambda_{1}, \lambda_{2}, \alpha_{2}\right) \in\left(\mathbb{C}^{*}\right)^{3}$ or $\left(\lambda_{1}, \lambda_{2}, \beta_{2}\right) \in\left(\mathbb{C}^{*}\right)^{3}$. Assume $\operatorname{Ind}\left(M_{1}\right)$ and $\operatorname{Ind}\left(M_{2}\right)$ are $\mathcal{H}$-modules defined by (2.2) for which $M_{1}$ and $M_{2}$ satisfy the conditions in Theorem 2.1. Then $\Omega\left(\lambda_{1}, \alpha_{1}, \beta_{1}\right) \otimes \operatorname{Ind}\left(M_{1}\right)$ and $\Omega\left(\lambda_{2}, \alpha_{2}, \beta_{2}\right) \otimes \operatorname{Ind}\left(M_{2}\right)$ are isomorphic as $\mathcal{H}$-modules if and only if $\left(\lambda_{1}, \alpha_{1}, \beta_{1}\right)=\left(\lambda_{2}, \alpha_{2}, \beta_{2}\right)$ and $\operatorname{Ind}\left(M_{1}\right) \cong \operatorname{Ind}\left(M_{2}\right)$ as $\mathcal{H}$-modules.

Proof. The "if" part is trivial. Now we prove the "only if" part. Let $\psi$ be an isomorphism from $\Omega\left(\lambda_{1}, \alpha_{1}, \beta_{1}\right) \otimes \operatorname{Ind}\left(M_{1}\right)$ to $\Omega\left(\lambda_{2}, \alpha_{2}, \beta_{2}\right) \otimes \operatorname{Ind}\left(M_{2}\right)$.

Choose a nonzero element $1 \otimes v \in \Omega\left(\lambda_{1}, \alpha_{1}, \beta_{1}\right) \otimes \operatorname{Ind}\left(M_{1}\right)$. Assume

$$
\psi(1 \otimes v)=\sum_{i=0}^{n} t^{i} \otimes v_{i}, \text { where } v_{i} \in \operatorname{Ind}\left(M_{2}\right) \text { with } v_{n} \neq 0
$$

There exists a positive integer $K=\max \left\{K(v), K\left(v_{i}\right) \mid i=0, \ldots, n\right\}$ such that $I_{m} \cdot v=$ $I_{m} \cdot v_{i}=L_{m} \cdot v=L_{m} \cdot v_{i}=0$ for all integers $m \geq K$ and $0 \leq i \leq n$.

Now we consider the following two cases.

Case 1. $\beta_{2} \in \mathbb{C}^{*}$. 
For any $m_{1}, m_{2} \geq K$, it follows from $\left(\lambda_{1}^{-m_{1}} I_{m_{1}}-\lambda_{1}^{-m_{2}} I_{m_{2}}\right) \cdot(1 \otimes v)=0$ that

$$
\begin{aligned}
0 & =\left(\lambda_{1}^{-m_{1}} I_{m_{1}}-\lambda_{1}^{-m_{2}} I_{m_{2}}\right) \cdot \psi(1 \otimes v) \\
& =\left(\lambda_{1}^{-m_{1}} I_{m_{1}}-\lambda_{1}^{-m_{2}} I_{m_{2}}\right) \cdot \sum_{i=0}^{n} t^{i} \otimes v_{i} \\
& =\sum_{i=0}^{n} \beta_{2}\left(\left(\frac{\lambda_{2}}{\lambda_{1}}\right)^{m_{1}}\left(t-m_{1}\right)^{i} \otimes v_{i}-\left(\frac{\lambda_{2}}{\lambda_{1}}\right)^{m_{2}}\left(t-m_{2}\right)^{i} \otimes v_{i}\right) .
\end{aligned}
$$

In particular, we have

$$
\left(\left(\frac{\lambda_{2}}{\lambda_{1}}\right)^{m_{1}}-\left(\frac{\lambda_{2}}{\lambda_{1}}\right)^{m_{2}}\right)\left(t^{n} \otimes v_{n}\right)=0 \quad \text { for all } m_{1}, m_{2} \geq K
$$

which forces $\lambda_{1}=\lambda_{2}$. Whence (4.2) can be rewritten as

$$
\sum_{i=0}^{n}\left(\left(t-m_{1}\right)^{i} \otimes v_{i}-\left(t-m_{2}\right)^{i} \otimes v_{i}\right)=0 \quad \text { for all } m_{1}, m_{2} \geq K
$$

Note from the above formula that $n=0$, since otherwise the coefficient $(-1)^{n}\left(1 \otimes v_{n}\right)$ of $m_{1}^{n}$ would be zero, yielding a contradiction $v_{n}=0$. Thus, by (4.1) there exists a linear bijection $\tau: \operatorname{Ind}\left(M_{1}\right) \rightarrow \operatorname{Ind}\left(M_{2}\right)$ such that

$$
\psi(1 \otimes v)=1 \otimes \tau(v) \text { for all } v \in \operatorname{Ind}\left(M_{1}\right) .
$$

From $\lambda_{1}=\lambda_{2}$ and $\psi\left(I_{m} \cdot(1 \otimes v)\right)=I_{m} \cdot \psi(1 \otimes v)$ for all $m \geq K$, it is easy to get $\beta_{1} \psi(1 \otimes v)=$ $\beta_{2}(1 \otimes \tau(v))$, which shows $\beta_{1}=\beta_{2}$. Since $\psi\left(I_{m} \cdot(1 \otimes v)\right)=I_{m} \cdot \psi(1 \otimes v)$ for all $m \in \mathbb{Z}$, we have $\psi\left(1 \otimes\left(I_{m} \cdot v\right)\right)=1 \otimes\left(I_{m} \cdot \tau(v)\right)$. Clearly,

$$
\tau\left(I_{m} \cdot v\right)=I_{m} \cdot \tau(v) \quad \text { for all } m \in \mathbb{Z}, v \in \operatorname{Ind}\left(M_{1}\right)
$$

For any $m_{1}, m_{2} \geq K$ and $m_{1} \neq m_{2}$, by

$$
\psi\left(\left(\lambda_{1}^{-m_{1}} L_{m_{1}}-\lambda_{1}^{-m_{2}} L_{m_{2}}\right) \cdot(1 \otimes v)\right)=\left(\lambda_{1}^{-m_{1}} L_{m_{1}}-\lambda_{1}^{-m_{2}} L_{m_{2}}\right) \cdot \psi(1 \otimes v),
$$

we can deduce that $\left(m_{2}-m_{1}\right) \alpha_{1} \psi(1 \otimes v)=\left(m_{2}-m_{1}\right) \alpha_{2}(1 \otimes \tau(v))$, which shows $\alpha_{1}=\alpha_{2}$. Using $\psi\left(L_{m} \cdot(1 \otimes v)\right)=L_{m} \cdot \psi(1 \otimes v)$ for all $m \geq K$, we can conclude that $\psi(t \otimes v)=t \otimes \tau(v)$. Therefore, we get $\psi\left(\left(L_{m} \cdot 1\right) \otimes v\right)=\left(L_{m} \cdot 1\right) \otimes \tau(v)$ for all $m \in \mathbb{Z}$. From

$$
\psi\left(L_{m} \cdot(1 \otimes v)\right)=L_{m} \cdot \psi(1 \otimes v) \text { for all } m \in \mathbb{Z},
$$

we can deduce that $\psi\left(1 \otimes\left(L_{m} \cdot v\right)\right)=1 \otimes\left(L_{m} \cdot \tau(v)\right)$. Hence,

$$
\tau\left(L_{m} \cdot v\right)=L_{m} \cdot \tau(v) \quad \text { for all } m \in \mathbb{Z}, v \in \operatorname{Ind}\left(M_{1}\right) .
$$

It is obvious that $\psi\left(C_{i} \cdot(1 \otimes v)\right)=C_{i} \cdot \psi(1 \otimes v)$ for $i=1,2,3, v \in \operatorname{Ind}\left(M_{1}\right)$, which implies $\tau\left(C_{i} \cdot v\right)=C_{i} \cdot \tau(v)$. This together with (4.3) and (4.4) show that $\psi$ is an $\mathcal{H}$-module isomorphism if $\beta_{2} \in \mathbb{C}^{*}$. 
Case 2. $\alpha_{2} \in \mathbb{C}^{*}$.

By the similar arguments as in the proof of [23, Theorem 2], we obtain that $\lambda_{1}=\lambda_{2}, \alpha_{1}=$ $\alpha_{2}$ and there exists a linear bijection $\tau: \operatorname{Ind}\left(M_{1}\right) \rightarrow \operatorname{Ind}\left(M_{2}\right)$ such that $\psi(1 \otimes v)=1 \otimes \tau(v)$ for all $v \in \operatorname{Ind}\left(M_{1}\right)$. At the same time, we get that $\tau\left(L_{m} \cdot v\right)=L_{m} \cdot \tau(v)$ for all $m \in$ $\mathbb{Z}, v \in \operatorname{Ind}\left(M_{1}\right)$. Since $\psi\left(I_{m} \cdot(1 \otimes v)\right)=I_{m} \cdot \psi(1 \otimes v)$ for all $m \geq K$, it is easy to see that $\beta_{1}=\beta_{2}$. Then from $\psi\left(I_{m} \cdot(1 \otimes v)\right)=I_{m} \cdot \psi(1 \otimes v)$ and $\psi\left(C_{i} \cdot(1 \otimes v)\right)=C_{i} \cdot \psi(1 \otimes v)$ for $i=1,2,3, m \in \mathbb{Z}$, we conclude that $\tau\left(I_{m} \cdot v\right)=I_{m} \cdot \tau(v)$ and $\tau\left(C_{i} \cdot v\right)=C_{i} \cdot \tau(v)$, respectively. Thus, $\operatorname{Ind}\left(M_{1}\right) \cong \operatorname{Ind}\left(M_{2}\right)$ as $\mathcal{H}$-modules for $\alpha_{2} \in \mathbb{C}^{*}$.

Combining the above two cases, we have the isomorphism criterion. This completes the proof of Theorem 4.1.

For any $c \in \mathbb{C}$, denote $\mathcal{I}_{c}$ by the (maximal) ideal of $\mathbb{C}\left[L_{0}\right]$ generated by $L_{0}-c$. For an $\mathcal{H}$-module $V$ and $n \in \mathbb{Z}$, set

$$
V_{n}=V / \mathcal{I}_{n} V, \mathcal{W}(V)=\oplus_{n \in \mathbb{Z}} V_{n}
$$

Then the vector space $\mathcal{W}(V)$ carries the structure of a weight (with respect to $\left.L_{0}\right) \mathcal{H}$-module under the following given actions (see [14,18]):

$$
\begin{gathered}
L_{m} \cdot\left(v+\mathcal{I}_{n} V\right)=L_{m} v+\mathcal{I}_{m+n} V \text { and } \\
I_{m} \cdot\left(v+\mathcal{I}_{n} V\right)=I_{m} v+\mathcal{I}_{m+n} V \quad \text { for } m, n \in \mathbb{Z} .
\end{gathered}
$$

Lemma 4.2. As $\mathcal{H}$-modules, $\mathcal{W}(\Omega(\lambda, \alpha, \beta)) \cong A(0,1-\alpha, \beta)$.

Proof. By the definition of $\mathcal{I}_{n}, \operatorname{dim}\left(\Omega(\lambda, \alpha, \beta) / \mathcal{I}_{n} \Omega(\lambda, \alpha, \beta)\right)=1$ for any $n \in \mathbb{Z}$. Take $v_{n}=1+\mathcal{I}_{n}(\Omega(\lambda, \alpha, \beta)) \in \Omega(\lambda, \alpha, \beta) / \mathcal{I}_{n}(\Omega(\lambda, \alpha, \beta))$. Then

$$
L_{m} v_{n}=\lambda^{m}(t-m \alpha)+\mathcal{I}_{m+n}(\Omega(\lambda, \alpha, \beta))=\lambda^{m}(n+m(1-\alpha)) v_{m+n},
$$

and $\quad I_{m} v_{n}=\lambda^{m} \beta+\mathcal{I}_{m+n}(\Omega(\lambda, \alpha, \beta))=\lambda^{m} \beta v_{m+n} \quad$ for any $m, n \in \mathbb{Z}$.

That is, $L_{m} w_{n}=(n+m(1-\alpha)) w_{m+n}$ and $I_{m} w_{n}=\beta w_{m+n}$, where $w_{n}=\lambda^{n} v_{n}$. This completes the proof of this lemma.

Proposition 4.3. We have the following isomorphism of $\mathcal{H}$-modules

$$
\mathcal{W}(\mathcal{M}(V, \Omega(\lambda, \alpha, \beta))) \cong \mathcal{M}(V, A(0,1-\alpha, \beta))
$$

Proof. Using (2.3), we have

$$
\mathcal{I}_{n}(\mathcal{M}(V, \Omega(\lambda, \alpha, \beta)))=\mathcal{I}_{n}(V \otimes \Omega(\lambda, \alpha, \beta))=V \otimes \mathcal{I}_{n}(\Omega(\lambda, \alpha, \beta)) \quad \text { for } n \in \mathbb{Z}
$$


Then it follows from this and Lemma 4.2 that

$$
\begin{aligned}
& \mathcal{W}(\mathcal{M}(V, \Omega(\lambda, \alpha, \beta)))=\bigoplus_{n \in \mathbb{Z}} \mathcal{M}(V, \Omega(\lambda, \alpha, \beta))_{n} \\
= & \bigoplus_{n \in \mathbb{Z}}\left(\mathcal{M}(V, \Omega(\lambda, \alpha, \beta)) / \mathcal{I}_{n}(\mathcal{M}(V, \Omega(\lambda, \alpha, \beta)))\right) \\
= & \left.\bigoplus_{n \in \mathbb{Z}}\left(V \otimes \Omega(\lambda, \alpha, \beta) / V \otimes \mathcal{I}_{n}(\Omega(\lambda, \alpha, \beta))\right) \quad(\text { by (2.3) })\right) \\
\cong & V \otimes \bigoplus_{n \in \mathbb{Z}}\left(\Omega(\lambda, \alpha, \beta) / \mathcal{I}_{n}(\Omega(\lambda, \alpha, \beta))\right)=V \otimes \mathcal{W}(\Omega(\lambda, \alpha, \beta)) \\
\cong & V \otimes A(0,1-\alpha, \beta)=\mathcal{M}(V, A(0,1-\alpha, \beta)) .
\end{aligned}
$$

Since $\mathcal{W}$ is a functor from the category of $\mathcal{H}$-modules to itself (see [18]), an $\mathcal{H}$-module isomorphism between $\mathcal{M}\left(V_{1}, \Omega\left(\lambda_{1}, \alpha_{1}, \beta_{1}\right)\right)$ and $\mathcal{M}\left(V_{2}, \Omega\left(\lambda_{2}, \alpha_{2}, \beta_{2}\right)\right)$ would imply $d_{1}=d_{2}$

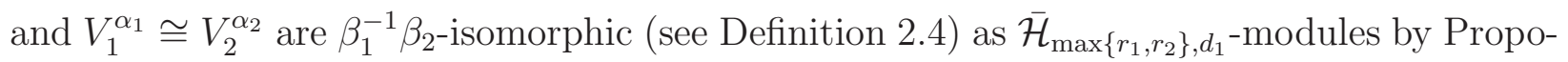
sition 4.3 and Theorem 2.5. So it is reasonable to include these into sufficient conditions for $\mathcal{M}\left(V_{1}, \Omega\left(\lambda_{1}, \alpha_{1}, \beta_{1}\right)\right)$ and $\mathcal{M}\left(V_{2}, \Omega\left(\lambda_{2}, \alpha_{2}, \beta_{2}\right)\right)$ being isomorphic. In fact, one more condition $\lambda_{1}=\lambda_{2}$ will be enough, as stated in the following result.

Theorem 4.4. Let $d_{i} \in\{0,1\}, r_{i} \in \mathbb{Z}_{+}, \lambda_{i}, \beta_{i} \in \mathbb{C}^{*}, \alpha_{i} \in \mathbb{C}$ and $V_{i}$ be an irreducible $\overline{\mathcal{H}}_{r_{i}, d_{i}{ }^{-}}$ module such that $\mathcal{M}\left(V_{i}, \Omega\left(\lambda_{i}, \alpha_{i}, \beta_{i}\right)\right)$ is irreducible for $i=1,2$. Then

$$
\mathcal{M}\left(V_{1}, \Omega\left(\lambda_{1}, \alpha_{1}, \beta_{1}\right)\right) \cong \mathcal{M}\left(V_{2}, \Omega\left(\lambda_{2}, \alpha_{2}, \beta_{2}\right)\right)
$$

as $\mathcal{H}$-modules if and only if $d_{1}=d_{2}, \lambda_{1}=\lambda_{2}$ and $V_{1}^{\alpha_{1}} \cong V_{2}^{\alpha_{2}}$ are $\beta_{1}^{-1} \beta_{2}$-isomorphic as $\overline{\mathcal{H}}_{\max \left\{r_{1}, r_{2}\right\}, d_{1}-\text { modules. }}$

Proof. Let

$$
\phi: \mathcal{M}\left(V_{1}, \Omega\left(\lambda_{1}, \alpha_{1}, \beta_{1}\right)\right) \rightarrow \mathcal{M}\left(V_{2}, \Omega\left(\lambda_{2}, \alpha_{2}, \beta_{2}\right)\right)
$$

be an isomorphism of $\mathcal{H}$-modules. By the remark before this theorem we know that $d_{1}=$ $d_{2}$ and that the linear map $\varphi: V_{1}^{\alpha_{1}} \rightarrow V_{2}^{\alpha_{2}}$ inducing from $\phi$ is a $\beta_{1}^{-1} \beta_{2}$-isomorphism of $\overline{\mathcal{H}}_{\text {max }\left\{r_{1}, r_{2}\right\}, d_{1}}$-modules. It remains to show $\lambda_{1}=\lambda_{2}$.

Take any $0 \neq w \in V_{1}$ and assume that $\phi(w \otimes 1)=\sum_{i} u_{i} \otimes t^{i} \in \mathcal{M}\left(V_{2}, \Omega\left(\lambda_{2}, \alpha_{2}, \beta_{2}\right)\right)$. Note on one hand that $\phi$ induces an $\mathcal{H}$-module isomorphism

$$
\phi_{A}: \mathcal{M}\left(V_{1}, A\left(0,1-\alpha_{1}, \beta_{1}\right)\right) \rightarrow \mathcal{M}\left(V_{2}, A\left(0,1-\alpha_{2}, \beta_{2}\right)\right)
$$


sending $w \otimes v_{n}$ to $\sum_{i}\left(\frac{\lambda_{2}}{\lambda_{1}}\right)^{n} n^{i} u_{i} \otimes v_{n}$ for any $n \in \mathbb{Z}$ by Lemma 4.2 and Proposition 4.3 , and on the other hand that $\phi_{A}\left(w \otimes v_{n}\right)=\varphi(w) \otimes v_{n}$ for any $n \in \mathbb{Z}$ (see [6, Theorem 4.1]). Thus, $\varphi(w)=\sum_{i}\left(\frac{\lambda_{2}}{\lambda_{1}}\right)^{n} n^{i} u_{i}$, which implies $\lambda_{1}=\lambda_{2}$ and $u_{i}=0$ if $i \neq 0$.

Conversely, let $\varphi: V_{1}^{\alpha_{1}} \rightarrow V_{2}^{\alpha_{2}}$ be a $\beta_{1}^{-1} \beta_{2}$-isomorphism of $\overline{\mathcal{H}}_{\max \left\{r_{1}, r_{2}\right\}, d_{1}}$-modules. One can check the linear map $\phi: \mathcal{M}\left(V_{1}, \Omega\left(\lambda, \alpha_{1}, \beta_{1}\right)\right) \rightarrow \mathcal{M}\left(V_{2}, \Omega\left(\lambda, \alpha_{2}, \beta_{2}\right)\right)$ sending $v \otimes f(t)$ to $\varphi(v) \otimes f(t)$ is an isomorphism of $\mathcal{H}$-modules.

\section{New irreducible modules}

In this section, we shall show that any one of $\Omega(\lambda, \alpha, \beta) \otimes \operatorname{Ind}(M)$ and $\mathcal{M}(V, \Omega(\lambda, \alpha, \beta))$ is not isomorphic to $\operatorname{Ind}(M)$ or the irreducible non-weight $\mathcal{H}$-modules defined in [6] and that $\Omega(\lambda, \alpha, \beta) \otimes \operatorname{Ind}(M)$ is not isomorphic to $\mathcal{M}\left(V, \Omega\left(\lambda^{\prime}, \alpha^{\prime}, \beta^{\prime}\right)\right)$.

For any $l, m \in \mathbb{Z}, s \in \mathbb{Z}_{+}$, define a sequence of operators $T_{l, m}^{(s)}$ as follows

$$
T_{l, m}^{(s)}=\sum_{i=0}^{s}(-1)^{s-i}\left(\begin{array}{l}
s \\
i
\end{array}\right) I_{l-m-i} I_{m+i} .
$$

Lemma 5.1. Let $\lambda \in \mathbb{C}^{*}, \alpha, \beta \in \mathbb{C}$ and $M$ be an irreducible $\mathcal{H}_{e}$-module satisfying the conditions in Theorem 2.1 and $V$ an irreducible $\overline{\mathcal{H}}_{r, d}$-module. Assume that $r^{\prime}$ is the maximal nonnegative integer such that $\bar{I}_{r^{\prime}+d} V \neq 0$. Then

(i) the action of $L_{m}$ for $m$ sufficiently large is not locally nilpotent on $\Omega(\lambda, \alpha, \beta) \otimes \operatorname{Ind}(M)$;

(ii) the action of $L_{m}$ for each $m \in \mathbb{Z}$ on $\mathcal{M}(V, \Omega(\lambda, \alpha, \beta))$ is not locally nilpotent;

(iii) $T_{l, m}^{\left(2 r^{\prime}+2 d\right)}$ is a linear isomorphism of $\mathcal{M}(V, \Omega(\lambda, \alpha, \beta))$ and $\widetilde{\mathcal{M}}(V, \gamma(t))$ for $l, m \in \mathbb{Z}$ and $\gamma(t) \in \mathbb{C}\left[t, t^{-1}\right]$

(iv) $T_{l, m}^{(s)}(s \geq 1)$ is locally nilpotent on $\Omega(\lambda, \alpha, \beta) \otimes \operatorname{Ind}(M)$ whenever $m \gg 0$ and $l \gg m$;

(v) $T_{l, m}^{(1)}$ acts nontrivially on $\Omega(\lambda, \alpha, \beta) \otimes \operatorname{Ind}(M)$ whenever $m \ll 0$ and $l \ll m$.

Proof. (i) follows from the local nilpotency of $L_{m}$ for $m$ sufficiently large on $\operatorname{Ind}(M)$ by Theorem 2.1 and its non-local nilpotency on $\Omega(\lambda, \alpha, \beta)$. (ii) and (iii) follows from [6, Lemma 3.3]. (iv) follows from an easy observation that

$$
T_{l, m}^{(s)} \Omega(\lambda, \alpha, \beta)=0 \quad \text { for } l, m \in \mathbb{Z}
$$

and $T_{l, m}^{(s)}$ is locally nilpotent on $\operatorname{Ind}(M)$ when $m \gg 0$ and $l \gg m$. Note when $m \ll 0$ and $l \ll m$ that $I_{l-m} \notin \mathcal{H}_{e}$ and $I_{m} \notin \mathcal{H}_{e}$. It follows from this and a direct computation that for $0 \neq 1 \otimes 1 \otimes v \in \Omega(\lambda, \alpha, \beta) \otimes \operatorname{Ind}(M)$ we have

$$
\begin{aligned}
T_{l, m}^{(1)}(1 \otimes 1 \otimes v)= & 1 \otimes\left(-\lambda^{m} \beta I_{l-m}-\lambda^{l-m} \beta I_{m}-I_{l-m} I_{m}+\right. \\
& \left.\lambda^{m+1} \beta I_{l-m-1}+\lambda^{l-m-1} \beta I_{m+1}+I_{l-m-1} I_{m+1}\right) \otimes v \neq 0 .
\end{aligned}
$$


So $T_{l, m}^{(1)}(\Omega(\lambda, \alpha, \beta) \otimes \operatorname{Ind}(M)) \neq 0$, proving $(\mathrm{v})$.

Let $d \in\{0,1\}, r \in \mathbb{Z}_{+}$and $V$ be an $\overline{\mathcal{H}}_{r, d}$-module. For any $\gamma(t)=\sum_{i} c_{i} t^{i} \in \mathbb{C}\left[t, t^{-1}\right]$, define the action of $\mathcal{H}$ on $V \otimes \mathbb{C}\left[t, t^{-1}\right]$ as follows

$$
\begin{aligned}
& L_{m} \circ\left(v \otimes t^{n}\right)=\left(L_{m}+\sum_{i} c_{i} I_{m+i}\right)\left(v \otimes t^{n}\right), \\
& I_{m} \circ\left(v \otimes t^{n}\right)=I_{m}\left(v \otimes t^{n}\right), \\
& C_{i} \circ\left(v \otimes t^{n}\right)=0 \quad \text { for } m, n \in \mathbb{Z}, v \in V \text { and } i=1,2,3 .
\end{aligned}
$$

Then $V \otimes \mathbb{C}\left[t, t^{-1}\right]$ carries the structure of an $\mathcal{H}$-module under the above given actions, which is denoted by $\widetilde{\mathcal{M}}(V, \gamma(t))$. Note that $\widetilde{\mathcal{M}}(V, \gamma(t))$ is a weight $\mathcal{H}$-module if and only if $\gamma(t) \in \mathbb{C}$ and also that the $\mathcal{H}$-module $\widetilde{\mathcal{M}}(V, \gamma(t))$ for $\gamma(t) \in \mathbb{C}\left[t, t^{-1}\right]$ is irreducible if and only if $V$ is irreducible (see [6]).

We are now ready to state the main result of this section.

Proposition 5.2. Let $d \in\{0,1\}, r, e \in \mathbb{Z}_{+}, \lambda \in \mathbb{C}^{*}, \alpha, \beta \in \mathbb{C}, M$ be an irreducible $\mathcal{H}_{e^{-}}$ module satisfying the conditions in Theorem 2.1 and $V$ an irreducible $\overline{\mathcal{H}}_{r, d}$-module. Then any of $\Omega(\lambda, \alpha, \beta) \otimes \operatorname{Ind}(M)$ and $\mathcal{M}(V, \Omega(\lambda, \alpha, \beta))$ is not isomorphic to $\operatorname{Ind}\left(M^{\prime}\right)$ for any irreducible $\mathcal{H}_{e}$-module $M^{\prime}$ satisfying the conditions in Theorem 2.1 or $\Omega\left(\lambda^{\prime}, \alpha^{\prime}, \beta^{\prime}\right)$ for any $\lambda^{\prime} \in \mathbb{C}^{*}, \alpha^{\prime}, \beta^{\prime} \in \mathbb{C}$, or $\widetilde{\mathcal{M}}(W, \gamma(t))$ for any $\overline{\mathcal{H}}_{r, d^{-}}$module $W$ and $\gamma(t)=\sum_{i} c_{i} t^{i} \in \mathbb{C}\left[t, t^{-1}\right]$; and $\Omega(\lambda, \alpha, \beta) \otimes \operatorname{Ind}(M)$ is not isomorphic to $\mathcal{M}\left(W, \Omega\left(\lambda^{\prime}, \alpha^{\prime}, \beta^{\prime}\right)\right)$

Proof. $\Omega(\lambda, \alpha, \beta) \otimes \operatorname{Ind}(M) \nsucceq \operatorname{Ind}\left(M^{\prime}\right)$ follows from Lemma 5.1(i) and Theorem 2.1, $\mathcal{M}(V$, $\Omega(\lambda, \alpha, \beta)) \varsubsetneqq \operatorname{Ind}\left(M^{\prime}\right)$ follows from Lemma 5.1(ii) and Theorem 2.1. $\Omega(\lambda, \alpha, \beta) \otimes \operatorname{Ind}(M) \not$ $\mathcal{M}\left(V, \Omega\left(\lambda^{\prime}, \alpha^{\prime}, \beta^{\prime}\right)\right)$ and $\Omega(\lambda, \alpha, \beta) \otimes \operatorname{Ind}(M) \nsucceq \widetilde{\mathcal{M}}(W, \gamma(t))$ follows from Lemma [5.1(iii) and (iv); $\Omega(\lambda, \alpha, \beta) \otimes \operatorname{Ind}(M) \nsucceq \Omega\left(\lambda^{\prime}, \alpha^{\prime}, \beta^{\prime}\right)$ follows from Lemma [5.1(v) and the fact $T_{l, m}^{(1)} \Omega(\lambda, \alpha, \beta)=0$ for $l, m \in \mathbb{Z}$. Finally, on the one hand, note that the restriction of $L_{0}-$ $\sum_{i} c_{i} I_{i}$ on $W \otimes t^{n}$ is the scalar $n$, namely, $L_{0}-\sum_{i} c_{i} I_{i}$ is semisimple on $\widetilde{\mathcal{M}}(W, \gamma(t))$; on the other hand, $L_{0}-\sum_{i} c_{i} I_{i}$ has no eigenvector in $\mathcal{M}(V, \Omega(\lambda, \alpha, \beta))$. Thus, $\mathcal{M}(V, \Omega(\lambda, \alpha, \beta)) \not$ $\widetilde{\mathcal{M}}(W, \gamma(t))$.

\section{Applications of $\Omega(\lambda, \alpha, \beta) \otimes \operatorname{Ind}(M)$}

Inspired by [17,23], we construct two classes of non-weight modules and then apply Theorem 3.1 to give certain conditions for these modules being irreducible.

For $\lambda \in \mathbb{C}^{*}$, we denote $\mathcal{H}_{\lambda}^{(0)}=\operatorname{span}_{\mathbb{C}}\left\{L_{m}-\lambda^{m} L_{0}, I_{0}, I_{m} \mid m \geq 1\right\}$ and $\mathcal{H}_{\lambda}^{(1)}=\operatorname{span}_{\mathbb{C}}\left\{L_{m}-\right.$ $\left.\lambda^{m-1} L_{1}, I_{n} \mid m \geq 2, n \geq 1\right\}$. It is easy to check that both $\mathcal{H}_{\lambda}^{(0)}$ and $\mathcal{H}_{\lambda}^{(1)}$ are Lie subalgebras 
of $\mathcal{H}$. For a fixed $R S=\left(r_{1}, r_{2}, s_{0}, s_{1}\right) \in \mathbb{C}^{4}$ and $P Q=\left(p_{2}, p_{3}, p_{4}, q_{1}, q_{2}\right) \in \mathbb{C}^{5}$, we define an $\mathcal{H}_{\lambda}^{(0)}$-action on $\mathbb{C}$ by

$$
\begin{aligned}
\left(L_{m}-\lambda^{m} L_{0}\right) \cdot 1 & =r_{m} \quad \text { for } m=1,2 ; \\
\left(L_{m}-\lambda^{m} L_{0}\right) \cdot 1 & =\lambda^{m-2}(m-1) r_{2}-\lambda^{m-1}(m-2) r_{1} \quad \text { for } m>2 ; \\
I_{m} \cdot 1 & =s_{m} \quad \text { for } m=0,1 ; \\
I_{m} \cdot 1 & =\lambda^{m-1} s_{1} \quad \text { for } m>1
\end{aligned}
$$

and an $\mathcal{H}_{\lambda}^{(1)}$-action on $\mathbb{C}$ by

$$
\begin{aligned}
\left(L_{m}-\lambda^{m-1} L_{1}\right) \cdot 1 & =p_{m} \quad \text { for } m=2,3,4 \\
\left(L_{m}-\lambda^{m-1} L_{1}\right) \cdot 1 & =\lambda^{m-4}(m-3) p_{4}-\lambda^{m-3}(m-4) p_{3} \quad \text { for } m>4 \\
I_{m} \cdot 1 & =q_{m} \quad \text { for } m=1,2 \\
I_{m} \cdot 1 & =\lambda^{m-2} q_{2} \quad \text { for } m>2
\end{aligned}
$$

It is straightforward to verify that under the given actions $\mathbb{C}$ is an $\mathcal{H}_{\lambda}^{(0)}$-module and also an $\mathcal{H}_{\lambda}^{(1)}$-module, denoted by $\mathbb{C}_{R S}$ and $\mathbb{C}_{P Q}$, respectively. For a fixed $\underline{y}=\left(y_{1}, y_{2}, y_{3}\right) \in \mathbb{C}^{3}$ and $\underline{z}=\left(z_{0}, z_{1}, z_{2}, z_{3}\right) \in \mathbb{C}^{4}$, we form the modules $\operatorname{Ind}_{\underline{y}, \lambda}\left(\mathbb{C}_{R S}\right)$ and $\operatorname{Ind}_{\underline{z}, \lambda}\left(\mathbb{C}_{P Q}\right)$ :

$$
\begin{aligned}
& \operatorname{Ind}_{\underline{y}, \lambda}\left(\mathbb{C}_{R S}\right)=\mathcal{U}(\mathcal{H}) \otimes_{\mathcal{U}\left(\mathcal{H}_{\lambda}^{(0)}\right)} \mathbb{C}_{R S} / \sum_{i=1}^{3}\left(C_{i}-y_{i}\right) \mathcal{U}(\mathcal{H}) \otimes_{\mathcal{U}\left(\mathcal{H}_{\lambda}^{(0)}\right)} \mathbb{C}_{R S}, \\
& \operatorname{Ind}_{\underline{z}, \lambda}\left(\mathbb{C}_{P Q}\right)=\mathcal{U}(\mathcal{H}) \otimes_{\mathcal{U}\left(\mathcal{H}_{\lambda}^{(1)}\right)} \mathbb{C}_{P Q} / \sum_{i=0}^{3}\left(C_{i}-z_{i}\right) \mathcal{U}(\mathcal{H}) \otimes_{\mathcal{U}\left(\mathcal{H}_{\lambda}^{(1)}\right)} \mathbb{C}_{P Q} .
\end{aligned}
$$

The following lemma is the key to proving the main results of this section, which generalizes [23, Lemma 6].

Lemma 6.1. Let $V$ be a cyclic $\mathcal{H}$-module with a basis

$$
\left\{I_{i-m}^{k_{i-m}} \cdots I_{i}^{k_{i}} L_{j-n}^{l_{j-n}} \cdots L_{j}^{l_{j}} \cdot v \mid m, n, k_{i}, \ldots, k_{i-m}, l_{j}, \ldots, l_{j-n} \in \mathbb{Z}_{+}\right\}
$$

where $0 \neq v \in V$ is a fixed vector, $i, j$ are fixed integers and $I_{p} \cdot v \in \mathbb{C} v, L_{q} \cdot v \in \mathbb{C} v$ for all integers $p>i, q>j$. Then for $(\lambda, \alpha) \in\left(\mathbb{C}^{*}\right)^{2}$ or $(\lambda, \beta) \in\left(\mathbb{C}^{*}\right)^{2}, \Omega(\lambda, \alpha, \beta) \otimes V$ is also a cyclic $\mathcal{H}$-module with a generator $1 \otimes v$ and a basis

$$
\mathcal{B}=\left\{I_{i-m}^{k_{i-m}} \cdots I_{i}^{k_{i}} L_{j-n}^{l_{j-n}} \cdots L_{j}^{l_{j}} L_{j+1}^{l_{j+1}} \cdot(1 \otimes v) \mid m, n, k_{i}, \ldots, k_{i-m}, l_{j+1}, l_{j}, \ldots, l_{j-n} \in \mathbb{Z}_{+}\right\} .
$$

Proof. Observe from (2.1) that $\Omega(\lambda, \alpha, \beta) \otimes V$ has a basis

$$
\mathcal{B}^{\prime}=\left\{t^{l_{j+1}} \otimes I_{i-m}^{k_{i-m}} \cdots I_{i}^{k_{i}} L_{j-n}^{l_{j-n}} \cdots L_{j}^{l_{j}} \cdot v \mid m, n, k_{i}, \ldots, k_{i-m}, l_{j+1}, l_{j}, \ldots, l_{j-n} \in \mathbb{Z}_{+}\right\}
$$


Now we define the following partial order " $\prec$ ” on $\mathcal{B}^{\prime}$

$$
t^{l_{j+1}} \otimes I_{i-m_{1}}^{k_{i-m_{1}}} \cdots I_{i}^{k_{i}} L_{j-n_{1}}^{l_{j-n_{1}}} \cdots L_{j}^{l_{j}} \cdot v \prec t^{q_{j+1}} \otimes I_{i-m_{2}}^{p_{i-m_{2}}} \cdots I_{i}^{p_{i}} L_{j-n_{2}}^{q_{j-n_{2}}} \cdots L_{j}^{q_{j}} \cdot v
$$

if and only if

$$
(l_{j}, \ldots, l_{j-n_{1}}, k_{i}, \ldots, k_{i-m_{1}}, \underbrace{0, \ldots, 0}_{m_{2}+n_{2}}, l_{j+1})<(q_{j}, \ldots, q_{j-n_{2}}, p_{i}, \ldots, p_{i-m_{2}}, \underbrace{0, \ldots, 0}_{m_{1}+n_{1}}, q_{j+1})
$$

in the lexicographical order, which is defined

$$
\left(a_{1}, \ldots, a_{\ell}\right)<\left(b_{1}, \ldots, b_{\ell}\right) \Longleftrightarrow \exists k>0 \text { such that } a_{i}=b_{i} \text { for all } i<k \text { and } a_{k}<b_{k} \text {. }
$$

Each element of $\mathcal{B}$ can be written as a linear combinations of elements in $\mathcal{B}^{\prime}$ :

$$
\begin{aligned}
& I_{i-m}^{k_{i-m}} \cdots I_{i}^{k_{i}} L_{j-n}^{l_{j-n}} \cdots L_{j}^{l_{j}} L_{j+1}^{l_{j+1}} \cdot(1 \otimes v) \\
= & \lambda^{(j+1) l_{j+1}} t^{l_{j+1}} \otimes I_{i-m}^{k_{i-m}} \cdots I_{i}^{k_{i}} L_{j-n}^{l_{j-n}} \cdots L_{j}^{l_{j}} \cdot v+\text { lower terms }(w . r . t \prec) .
\end{aligned}
$$

This shows that the transition matrix from $\mathcal{B}^{\prime}$ to $\mathcal{B}$ is upper triangular with diagonal entries nonzero. Thus, $\mathcal{B}$ is a basis of $\Omega(\lambda, \alpha, \beta) \otimes V$ and the lemma follows.

Now we are ready to give some conditions under which $\operatorname{Ind}_{\underline{y}, \lambda}\left(\mathbb{C}_{R S}\right)$ is irreducible.

Theorem 6.2. Let $\lambda \in \mathbb{C}^{*}, \underline{y}=\left(y_{1}, y_{2}, y_{3}\right) \in \mathbb{C}^{3}, R S=\left(r_{1}, r_{2}, s_{0}, s_{1}\right) \in \mathbb{C}^{4}$ with $y_{3}=0$. Assume $\operatorname{Ind}_{\underline{y}, \lambda}\left(\mathbb{C}_{R S}\right)$ is defined as in (6.3). Then

(i) $\operatorname{Ind}_{\underline{y}, \lambda}\left(\mathbb{C}_{R S}\right) \cong \Omega(\lambda, \alpha, \beta) \otimes V$, where $V$ is the classical Verma module described in Example 3.2 (i) and $\alpha, \beta, h, d_{i}$ for $i=0,1,2,3$ are defined as

$$
\begin{aligned}
& \alpha=\lambda^{-2}\left(\lambda r_{1}-r_{2}\right), \beta=\lambda^{-1} s_{1}, h=\lambda^{-2}\left(r_{2}-2 \lambda r_{1}\right), \\
& d_{0}=s_{0}-\lambda^{-1} s_{1}, d_{3}=y_{3}=0, d_{i}=y_{i} \quad \text { for } i=1,2 .
\end{aligned}
$$

(ii) $\operatorname{Ind}_{\underline{y}, \lambda}\left(\mathbb{C}_{R S}\right)$ is irreducible if $s_{0}-\lambda^{-1} s_{1}+(n-1) y_{2} \neq 0$ for all $n \in \mathbb{Z} \backslash\{0\}$, and either $r_{2} \neq$ $\lambda r_{1}$ or $s_{1} \neq 0$.

Proof. (i) Let $\alpha, \beta, h, d_{i} \in \mathbb{C}$ for $i=0,1,2,3$ as in (6.5). Then

$$
\begin{aligned}
& r_{1}=-\lambda(\alpha+h), r_{2}=-\lambda^{2}(2 \alpha+h), s_{1}=\lambda \beta, \\
& s_{0}=d_{0}+\beta, y_{3}=d_{3}=0, y_{i}=d_{i} \text { for } i=1,2 .
\end{aligned}
$$

Denote $v=1+J_{1} \in V$. By Lemma 6.1 and the structure of $V, \Omega(\lambda, \alpha, \beta) \otimes V$ is a cyclic module with a generator $1 \otimes v$ and has a basis

$$
\mathcal{B}_{1}=\left\{I_{-n}^{l_{-n}} \cdots I_{-1}^{l_{-1}} L_{-m}^{k_{-m}} \cdots L_{-1}^{k_{-1}} L_{0}^{k_{0}} \cdot(1 \otimes v) \mid m, n, k_{-m}, \ldots, k_{-1}, k_{0}, l_{-n}, \ldots, l_{-1} \in \mathbb{Z}_{+}\right\} .
$$


By Theorem 3.1 and the fact that $d_{3}=0, \Omega(\lambda, \alpha, \beta) \otimes V$ is irreducible if $d_{0}+(n-1) d_{2} \neq 0$ for all $n \in \mathbb{Z} \backslash\{0\}$ and either $\alpha \in \mathbb{C}^{*}$ or $\beta \in \mathbb{C}^{*}$.

In $\Omega(\lambda, \alpha, \beta) \otimes V$, we can compute that

$$
\begin{aligned}
\left(L_{m}-\lambda^{m} L_{0}\right) \cdot(1 \otimes v) & =-\lambda^{m}(m \alpha+h)(1 \otimes v)=r_{m}(1 \otimes v) \quad \text { for } m=1,2 ; \\
\left(L_{m}-\lambda^{m} L_{0}\right) \cdot(1 \otimes v) & =-\lambda^{m}(m \alpha+h)(1 \otimes v) \\
& =\left(\lambda^{m-2}(m-1) r_{2}-\lambda^{m-1}(m-2) r_{1}\right)(1 \otimes v) \quad \text { for } m>2 ; \\
I_{m} \cdot(1 \otimes v) & =\left(\lambda^{m} \beta+\delta_{m, 0} d_{0}\right)(1 \otimes v)=s_{m}(1 \otimes v) \quad \text { for } m=0,1 ; \\
I_{m} \cdot(1 \otimes v) & =\lambda^{m} \beta(1 \otimes v)=\lambda^{m-1} s_{1}(1 \otimes v) \quad \text { for } m>1
\end{aligned}
$$

and $C_{i} \cdot(1 \otimes v)=d_{i}(1 \otimes v)=y_{i}(1 \otimes v)$ for $i=1,2,3$. Comparing (6.1) with (6.6), we deduce that there exists an $\mathcal{H}$-module homomorphism (epimorphism)

$$
\tau: \operatorname{Ind}_{\underline{y}, \lambda}\left(\mathbb{C}_{R S}\right) \rightarrow \Omega(\lambda, \alpha, \beta) \otimes V
$$

which is uniquely determined by $\tau(\overline{1})=1 \otimes v$ with

$$
\overline{1}:=1 \otimes 1+\sum_{i=1}^{3}\left(C_{i}-y_{i}\right) \mathcal{U}(\mathcal{H}) \otimes_{\mathcal{U}\left(\mathcal{H}_{\lambda}^{(0)}\right)} \mathbb{C}_{R S} \in \operatorname{Ind}_{\underline{y}, \lambda}\left(\mathbb{C}_{R S}\right)
$$

Clearly, $\operatorname{Ind}_{\underline{y}, \lambda}\left(\mathbb{C}_{R S}\right)$ has a basis

$$
\mathcal{B}_{2}=\left\{I_{-n}^{l_{-n}} \cdots I_{-1}^{l_{-1}} L_{-m}^{k_{-m}} \cdots L_{-1}^{k_{-1}} L_{0}^{k_{0}} \cdot \overline{1} \mid m, n, k_{-m}, \ldots, k_{-1}, k_{0}, l_{-n}, \ldots, l_{-1} \in \mathbb{Z}_{+}\right\} .
$$

Since $\left.\tau\right|_{\mathcal{B}_{2}}: \mathcal{B}_{2} \rightarrow \mathcal{B}_{1}$ is a bijection, $\tau: \operatorname{Ind}_{\underline{y}, \lambda}\left(\mathbb{C}_{R S}\right) \rightarrow \Omega(\lambda, \alpha, \beta) \otimes V$ is an isomorphism. Hence, (i) holds.

(ii) By (i) and Theorem [3.1, $\operatorname{Ind}_{\underline{y}, \lambda}\left(\mathbb{C}_{R S}\right)$ is irreducible if and only if $\Omega(\lambda, \alpha, \beta) \otimes V$ is irreducible. But Examples [3.2(i), $\Omega(\lambda, \alpha, \beta) \otimes V$ is irreducible if $d_{0}+(n-1) d_{2} \neq 0$ for all $n \in$ $\mathbb{Z} \backslash\{0\}$ and either $\alpha \neq 0$ or $\beta \neq 0$ by noting $d_{3}=0$. Thus by (6.5) $\operatorname{Ind}_{\underline{y}, \lambda}\left(\mathbb{C}_{R S}\right)$ is irreducible if $s_{0}-\lambda^{-1} s_{1}+(n-1) y_{2} \neq 0$ for all $n \in \mathbb{Z} \backslash\{0\}$ and either $r_{2} \neq \lambda r_{1}$ or $s_{1} \neq 0$. Theorem 6.2 is proved.

While the irreducible conditions of $\operatorname{Ind}_{\underline{z}, \lambda}\left(\mathbb{C}_{P Q}\right)$ can be given as follows.

Theorem 6.3. Let $\lambda \in \mathbb{C}^{*}, \underline{z}=\left(z_{0}, z_{1}, z_{2}, z_{3}\right) \in \mathbb{C}^{4}, P Q=\left(p_{2}, p_{3}, p_{4}, q_{1}, q_{2}\right) \in \mathbb{C}^{5}$ with $z_{3}=0$. Assume $\operatorname{Ind}_{\underline{z}, \lambda}\left(\mathbb{C}_{P Q}\right)$ is defined as in (6.4). Then

(i) $\operatorname{Ind}_{\underline{z}, \lambda}\left(\mathbb{C}_{P Q}\right) \cong \Omega(\lambda, \alpha, \beta) \otimes V$, where $V$ is the classical Whittaker module described in Example 3.2 (ii) and $\alpha, \beta, \lambda_{1}, \lambda_{2}, \mu_{1}, e_{i}$ for $i=0,1,2,3$ are defined as

$$
\begin{aligned}
& \alpha=\lambda^{-4}\left(\lambda p_{3}-p_{4}\right), \beta=\lambda^{-2} q_{2}, \lambda_{1}=\lambda^{-3}\left(2 p_{4}-3 \lambda p_{3}\right), \\
& \lambda_{2}=\lambda^{-2}\left(p_{4}-2 \lambda p_{3}+\lambda^{2} p_{2}\right), \mu_{1}=q_{1}-\lambda^{-1} q_{2}, \\
& e_{3}=z_{3}=0, e_{0}=z_{0}-\lambda^{-2} q_{2}, e_{i}=z_{i} \quad \text { for } i=1,2
\end{aligned}
$$


(ii) $\operatorname{Ind}_{\underline{z}, \lambda}\left(\mathbb{C}_{P Q}\right)$ is irreducible if $z_{0}+(n-1) z_{2} \neq 0$ for all $n \in \mathbb{Z} \backslash\{0\}, \lambda q_{1} \neq q_{2}$ and either $p_{4} \not \hat{f} \lambda p_{3}$ or $q_{2} \neq 0$,

Proof. (i) Let $\alpha, \beta, \lambda_{1}, \lambda_{2}, \mu_{1} \in \mathbb{C}$ be as in (6.7). Then we have

$$
\begin{aligned}
& p_{2}=\lambda_{2}-\lambda \lambda_{1}-\lambda^{2} \alpha, p_{3}=-\lambda^{2}\left(\lambda_{1}+2 \lambda \alpha\right), p_{4}=-\lambda^{3}\left(\lambda_{1}+3 \lambda \alpha\right), \\
& q_{1}=\mu_{1}+\lambda \beta, q_{2}=\lambda^{2} \beta, z_{3}=e_{3}=0, z_{0}=e_{0}+\beta, z_{i}=e_{i} \text { for } i=1,2 .
\end{aligned}
$$

Denote $v=1+J_{2} \in V$. Clearly, $\mathcal{H}_{+} \cdot v \in \mathbb{C} v$. Since $V$ has a basis

$$
\left\{I_{-n}^{l_{-n}} \cdots I_{-1}^{l_{-1}} L_{-m}^{k_{-m}} \cdots L_{-1}^{k_{-1}} L_{0}^{k_{0}} \cdot v \mid m, n, k_{-m}, \ldots, k_{0}, l_{-n}, \ldots, l_{-1} \in \mathbb{Z}_{+}\right\}
$$

using Lemma 6.1, we see that $\Omega(\lambda, \alpha, \beta) \otimes V$ is cyclic with a generator $1 \otimes v$ and has a basis

$$
\mathcal{B}_{1}=\left\{I_{-n}^{l_{-n}} \cdots I_{-1}^{l_{-1}} L_{-m}^{k_{-m}} \cdots L_{0}^{k_{0}} L_{1}^{k_{1}} \cdot(1 \otimes v) \mid m, n, k_{-m}, \ldots, k_{0}, k_{1}, l_{-n}, \ldots, l_{-1} \in \mathbb{Z}_{+}\right\}
$$

By Theorem 3.1 and the fact that $e_{3}=0, \Omega(\lambda, \alpha, \beta) \otimes V$ is irreducible if $e_{0}+(n-1) e_{2} \neq 0$ for all $n \in \mathbb{Z} \backslash\{0\}, \mu_{1} \neq 0$ and either $\alpha \in \mathbb{C}^{*}$ or $\beta \in \mathbb{C}^{*}$.

In $W$, we can compute that

$$
\begin{aligned}
\left(L_{m}-\lambda^{m-1} L_{1}\right) \cdot(1 \otimes v) & =\left(\lambda^{m}(1-m) \alpha-\lambda^{m-1} \lambda_{1}+\delta_{2-m, 0} \lambda_{2}\right)(1 \otimes v) \\
& =p_{m}(1 \otimes v) \quad \text { for } m=2,3,4 ; \\
\left(L_{m}-\lambda^{m-1} L_{1}\right) \cdot(1 \otimes v) & =\left(\lambda^{m}(1-m) \alpha-\lambda^{m-1} \lambda_{1}\right)(1 \otimes v) \\
& =\left(\lambda^{m-4}(m-3) p_{4}-\lambda^{m-3}(m-4) p_{3}\right)(1 \otimes v) \quad \text { for } m>4 ; \\
I_{m} \cdot(1 \otimes v) & =\left(\lambda^{m} \beta+\delta_{1-m, 0} \mu_{1}\right)(1 \otimes v)=q_{m}(1 \otimes v) \quad \text { for } m=1,2 ; \\
I_{m} \cdot(1 \otimes v) & =\lambda^{m} \beta(1 \otimes v)=\lambda^{m-2} q_{2}(1 \otimes v) \quad \text { for } m>2
\end{aligned}
$$

and $C_{i} \cdot(1 \otimes v)=\left(e_{i}+\delta_{i, 0} \beta\right)(1 \otimes v)=z_{i}(1 \otimes v)$ for $i=0,1,2,3$. Comparing (6.2) with (6.8), there exists an $\mathcal{H}$-module homomorphism (epimorphism)

$$
\tau: \operatorname{Ind}_{\underline{z}, \lambda}\left(\mathbb{C}_{P Q}\right) \rightarrow \Omega(\lambda, \alpha, \beta) \otimes V
$$

which uniquely determined by $\tau(\overline{1})=1 \otimes v$ with

$$
\overline{1}:=1 \otimes 1+\sum_{i=0}^{3}\left(C_{i}-z_{i}\right) \mathcal{U}(\mathcal{H}) \otimes_{\mathcal{U}\left(\mathcal{H}_{\lambda}^{(1)}\right)} \mathbb{C}_{P Q} \in \operatorname{Ind}_{\underline{z}, \lambda}\left(\mathbb{C}_{P Q}\right)
$$

It is clear that $\operatorname{Ind}_{\underline{z}, \lambda}\left(\mathbb{C}_{P Q}\right)$ has a basis

$$
\mathcal{B}_{2}=\left\{I_{-n}^{l_{-n}} \cdots I_{-1}^{l_{-1}} L_{-m}^{k_{-m}} \cdots L_{0}^{k_{0}} L_{1}^{k_{1}} \cdot \overline{1} \mid m, n, k_{-m}, \ldots, k_{0}, k_{1}, l_{-n}, \ldots, l_{-1} \in \mathbb{Z}_{+}\right\}
$$


Since $\left.\tau\right|_{\mathcal{B}_{2}}: \mathcal{B}_{2} \rightarrow \mathcal{B}_{1}$ is a bijection, $\tau: \operatorname{Ind}_{\underline{z}, \lambda}\left(\mathbb{C}_{P Q}\right) \rightarrow \Omega(\lambda, \alpha, \beta) \otimes V$ is an isomorphism. This completes the proof of part (i).

(ii) By (i) and Theorem [3.1, $\operatorname{Ind}_{\underline{z}, \lambda}\left(\mathbb{C}_{P Q}\right)$ is irreducible if and only if $\Omega(\lambda, \alpha, \beta) \otimes V$ is irreducible. But by Examples $3.2($ ii $), \Omega(\lambda, \alpha, \beta) \otimes V$ is irreducible if $e_{0}+(n-1) e_{2} \neq$ 0 for all $n \in \mathbb{Z} \backslash\{0\}, \mu_{1} \neq 0$ and either $\alpha \neq 0$ or $\beta \neq 0$ by noting $e_{3}=0$. Thus by (6.7) $\operatorname{Ind}_{\underline{z}, \lambda}\left(\mathbb{C}_{P Q}\right)$ is irreducible if $z_{0}+(n-1) z_{2} \neq 0$ for all $n \in \mathbb{Z} \backslash\{0\}, \lambda q_{1} \neq q_{2}$ and either $p_{4} \neq \lambda p_{3}$ or $q_{2} \neq 0$.

\section{Acknowledgments}

This work was supported by NSF grant Nos. 11431010, 11371278, 11501417, 11671247, Innovation Program of Shanghai Municipal Education Commission.

\section{References}

[1] E. Arbarello, C. DeConcini, V. G. Kac, C. Procesi, Moduli spaces of curves and representation theory, Commun. Math. Phys. 117 (1988), 1-36.

[2] Y. Billig, Representations of the twisted Heisenberg-Virasoro algebra at level zero, Canad. Math. Bull. 46 (2003), 529-537.

[3] H. Chen, X. Guo, New simple modules for the Heisenberg-Virasoro algebra, J. Algebra 390 (2013), 77-86.

[4] H. Chen, X. Guo, Non-weight modules over the Heisenberg-Virasoro algebra and the $W$ algebra $W(2,2)$, J. Algebra Appl. 16 (2017), 1750097.

[5] H. Chen, X. Guo, K. Zhao, Tensor product weight modules over the Virasoro algebra, J. Lond. Math. Soc. 88 (2013), 829-844.

[6] H. Chen, J. Han, Y. Su, A class of simple weight modules over the twisted HeisenbergVirasoro algebra, J. Math. Phys. 57 (2016), 101705, 7 pp.

[7] H. Chen, J. Li, Left-symmetric algebra structures on the twisted Heisenberg-Virasoro algebra, Sci. China Math. 57 (2014), 469-476.

[8] J. Han, Q. Chen, Y. Su, Modules over the algebra Vir(a,b), Linear Algebra Appl. 515 (2017), 11-23.

[9] I. Kaplansky, L. J. Santharoubane, Harish-Chandra modules over the Virasoro algebra, Math. Sci. Res. Inst. Publ. 4 (1985), Springer, New York, 217-231.

[10] D. Liu, Y. Pei, L. Zhu, Lie bialgebra structures on the twisted Heisenberg-Virasoro algebra, J. Algebra 359 (2012), 35-48.

[11] D. Liu, C. Jiang, Harish-Chandra modules over the twisted Heisenberg-Virasoro algebra, J. Math. Phys. 49 (2008), 012901, 13 pp. 
[12] D. Liu, Y. Wu, L. Zhu, Whittaker modules for the twisted Heisenberg-Virasoro algebra, J. Math. Phys. 51 (2010), 023524, 12 pp.

[13] R. Lü, K. Zhao, Irreducible Virasoro modules from irreducible Weyl modules, J. Algebra 414 (2014), 271-287.

[14] G. Liu, Y. Zhao, Generalized polynomial modules over the Virasoro algebra, Proc. Amer. Math. Soc. 144 (2016), 5103-5112.

[15] R. Lü, K. Zhao, Classification of irreducible weight modules over the twisted HeisenbergVirasoro algebra, Commun. Contemp. Math. 12 (2010), 183-205.

[16] O. Mathieu, Classification of Harish-Chandra modules over the Virasoro Lie algebra, Invent. Math. 107 (1992), 225-234.

[17] V. Mazorchuk, E. Weisner, Simple Virasoro modules induced from codimension one subalgebras of the positive part, Proc. Amer. Math. Soc. 142 (2014), 3695-3703.

[18] J. Nilsson, $U(\mathfrak{h})$-free modules and coherent families, J. Pure Appl. Algebra 220 (2016), $1475-1488$.

[19] G. Radobolja, Subsingular vectors in Verma modules, and tensor product of weight modules over the twisted Heisenberg-Virasoro algebra and W(2,2) algebra, J. Math. Phys. 54 (2013), 071701, 24 pp.

[20] R. Shen, C. Jiang, The derivation algebra and automorphism group of the twisted Heisenberg-Virasoro algebra, Comm. Algebra 34 (2006), 2547-2558.

[21] R. Shen, Q. Jiang, Y. Su, Verma modules over the generalized Heisenberg-Virasoro algebra, Comm. Algebra 04 (2008), 1464-1473.

[22] R. Shen, Y. Su, Classification of irreducible weight modules with a finite-dimensional weight space over twisted Heisenberg-Virasoro algebra, Acta Math. Sin. (E. S.) 23 (2007), 189-192.

[23] H. Tan, K. Zhao, Irreducible Virasoro modules from tensor products, Ark. Mat. 54 (2016), 181-200.

[24] H. Tan, K. Zhao, Irreducible Virasoro modules from tensor products (II), J. Algebra 394 (2013), 357-373.

[25] H. Zhang, A class of representations over the Virasoro algebra, J. Algebra 190 (1997), 1-10. 